\title{
ESTRATEGIAS DE DIFUSIÓN DE UNA REVISTA CIENTÍFICA. UN EXPERIMENTO CON EL PROFESIONAL DE LA INFORMACIÓN
}

\section{Sergi Cortiñas-Rovira y Xavier Ramon-Vegas}
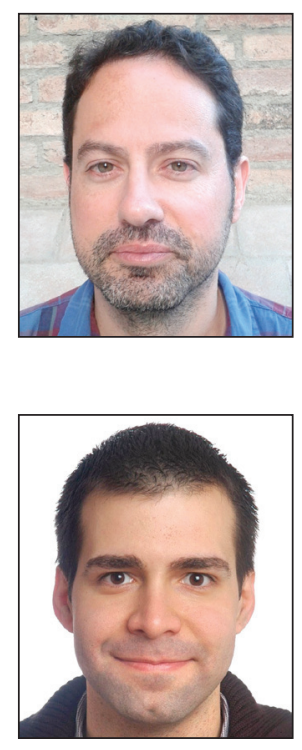

Sergi Cortiñas-Rovira es profesor del Depto. de Comunicación de la Univ. Pompeu Fabra (UPF). Doctor en comunicación social por la UPF, licenciado en ciencias químicas por la Univ. de Barcelona $(U B)$ y periodismo por la UPF, investiga sobre divulgación de la ciencia. Dirige el proyecto competitivo I+D+i El periodismo científico en España y las nuevas tecnologías de la información (TIC): mapa de situación y propuesta de actuación para mejorar los procesos comunicativos, del que forma parte este artículo (Mo de Economía y Competitividad, Ref.: CSO2011-25969). http://orcid.org/0000-0002-7252-5418

sergi.cortinas@upf.edu

Xavier Ramon-Vegas es profesor e investigador del Departamento de Comunicación de la Universitat Pompeu Fabra. Doctorando en comunicación, es máster en estudios avanzados en comunicación social y licenciado en comunicación audiovisual y periodismo por la UPF.

http://orcid.org/0000-0002-4478-5626

xavier.ramon@upf.edu

Departamento de Comunicación, Universitat Pompeu Fabra Roc Boronat, 138. 08018 Barcelona http://www.upf.edu/decom/es

\section{Resumen}

Partiendo de los fundamentos teóricos sobre el papel de los gabinetes de prensa en la transmisión de contenidos de las revistas científicas, se analiza un experimento realizado entre noviembre de 2011 y noviembre de 2012 para difundir los contenidos de El profesional de la información. El documento detalla la propuesta metodológica, las fuentes utilizadas, los focos de envío y la selección y edición de la información. Se ahonda además en las características de los impactos obtenidos. Finalmente se exponen propuestas futuras para potenciar la transmisión y monitorización de los contenidos científicos mediante las notas de prensa y las redes sociales.

\section{Palabras clave}

Difusión del conocimiento científico, Revistas científicas, Gabinetes de comunicación, Notas de prensa, Experimento, El profesional de la información.

Title: Strategies for disseminating a scientific journal. An experiment with El profesional de la información

\begin{abstract}
Starting from the theoretical foundations of the role of press offices in the transmission of the content of scientific journals, the article analyses an experiment carried out between November 2011 and November 2012 with El profesional de la información. The methodological approach, the sources, the dissemination targets and the selection and edition of the information are discussed. Moreover, an in-depth analysis of the characteristics of the achieved impacts is presented. Finally, future research is suggested to enhance the transmission and monitoring of scientific content through press releases and social networking sites.
\end{abstract}

\section{Keywords}

Dissemination of scientific knowledge, Scientific journals, Communication offices, Press releases, Experiment, El profesional de la información.

Cortiñas-Rovira, Sergi; Ramon-Vegas, Xavier (2013). “Estrategias de difusión de una revista científica. Un experimento con El profesional de la información". El profesional de la información, septiembre-octubre, v. 22, n. 5, pp. $405-414$.

http://dx.doi.org/10.3145/epi.2013.sep.05 


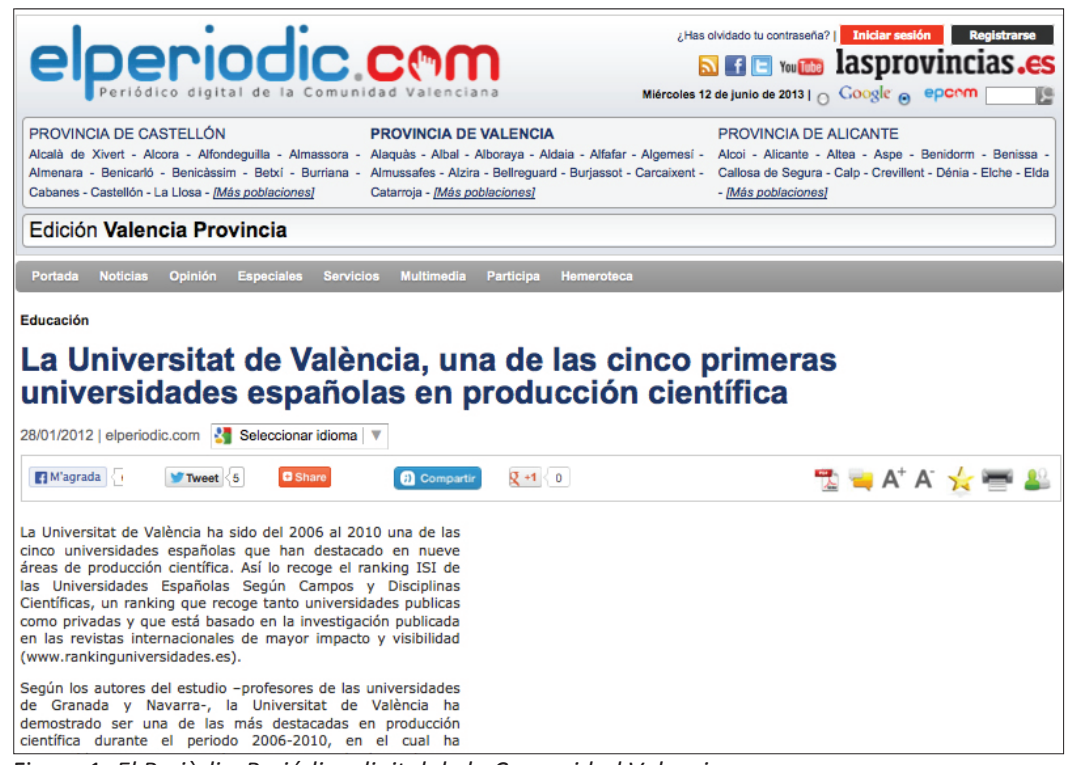

Figura 1. El Periòdic. Periódico digital de la Comunidad Valenciana https://www.facebook.com/elprofesionaldelainformacion

http://twitter.com/revista_EPI

\section{Experimento. Difusión de contenidos de EPI}

\subsection{Definición y propuesta metodo- lógica}

Con el objetivo de captar el interés informativo (Sissons, 2012) e intentar lograr impactos para la visibilidad de la revista, se realizó durante el período de 13 meses comprendido entre noviembre de 2011 y noviembre de 2012 un experimento de campo (Priest, 2009, p. 83). Este método se basa en "manipular la variable independiente para determinar si tiene una influencia en la variable dependiente" (Bryman, 2012, p. 50). En este caso se tomó como variable independiente una muestra

\section{El papel de los gabinetes de prensa en la difusión de contenidos científicos}

La actividad de los gabinetes de prensa en España cuenta con una dilatada trayectoria (Almansa-Martínez, 2011, pp. 36-53). Sin embargo la difusión de los contenidos científicos por parte de los medios ha sido escasa. Por un lado "las noticias científicas tan sólo aparecen en los medios de comunicación cuando el descubrimiento está avalado por una publicación de impacto o por un premio de prestigio" (Domínguez-Romero, 2002, p. 50). Por otra parte, se percibe una baja visibilidad de las investigaciones españolas frente a los contenidos extranjeros (Nieto; Vendrell, 2001), así como la minimización de campos fuera de la medicina, agricultura o medio ambiente (Elías, 2002).

En el ecosistema comunicativo actual, las revistas científicas deben aprovechar todas las fórmulas que ofrece el entorno digital para gestionar eficientemente las relaciones con la prensa

Pese a todo, en el ecosistema comunicativo actual, marcado por la instantaneidad y la superabundancia informativa (Fernández-Asenjo; De-la-Torre-Alfaro, 2009), las revistas científicas deben aprovechar todas las fórmulas que ofrece el entorno digital para gestionar eficientemente las relaciones con la prensa (Almansa-Martínez, 2011; García-Orosa, 2009). La publicación en medios y sitios web institucionales es clave en la estrategia de promoción de los artículos científicos, junto con la difusión en repositorios y redes sociales (Baiget; Torres-Salinas, 2013, pp. 86-89). En esta línea la revista El profesional de la información (EPI) realiza una tarea de comunicación intensiva en su web y blog, así como en las redes sociales Facebook y Twitter. No obstante, potenciar los contenidos de EPI entre medios e instituciones era, y en cierta medida sigue siendo, un reto pendiente de superar. http://www.elprofesionaldelainformacion.com/notas de artículos de EPI. Mediante el experimento los investigadores 1) diseñaron una estrategia de difusión, 2) transformaron los contenidos en notas de prensa y 3) recogieron datos sobre el impacto que éstas tuvieron sobre la variable dependiente, que en este experimento es el espacio informativo de los medios e instituciones (Campbell; Stanley, 1966; Priest, 2009; Webster; Sell, 2007).

\subsection{Creación de un marco para la difusión de conte- nidos}

En diciembre de 2011 se elaboró una primera base de datos de medios, instituciones y periodistas. Se tomó como punto de partida la Agenda de comunicación 2011, editada por la Secretaría de Estado de Comunicación (Ministerio de la Presidencia del Gobierno de España) ${ }^{1}$. Se seleccionó un total de 1.120 direcciones relevantes de correo electrónico ${ }^{2}$ de medios de diferentes soportes (prensa escrita, radio, televisión

Tabla 1. Destinatarios de la difusión considerados en el estudio

\begin{tabular}{|c|c|}
\hline 1 & Periódicos nacionales y autonómicos de información general \\
\hline 2 & Prensa nativa online \\
\hline 3 & $\begin{array}{l}\text { Revistas especializadas en tecnología, comunicación, cultura } \\
\text { y arte }\end{array}$ \\
\hline 4 & Revistas de información general \\
\hline 5 & Revistas especializadas en ciencia \\
\hline 6 & Agencias de noticias \\
\hline 7 & Emisoras de radio y televisión \\
\hline 8 & $\begin{array}{l}\text { Listas de distribución científica (Agencia SINC, Associació } \\
\text { Catalana de Comunicació Científica, Incyt, etc.) }\end{array}$ \\
\hline 9 & $\begin{array}{l}\text { Portales de comunicación (Portal de la comunicació, CSIC, } \\
\text { Noticom, etc.). }\end{array}$ \\
\hline 10 & Expertos y académicos \\
\hline 11 & $\begin{array}{l}\text { Departamentos de prensa y gabinetes de comunicación de } \\
\text { universidades }\end{array}$ \\
\hline 12 & $\begin{array}{l}\text { Departamentos de comunicación de instituciones, gobiernos } \\
\text { y empresas }\end{array}$ \\
\hline 13 & Autores de blogs \\
\hline
\end{tabular}


e internet), tipo (generalistas y especializados) y ámbitos geográficos (nacionales, autonómicos y locales). Posteriormente se actualizó la base de datos a raíz de la publicación de la Agenda de la comunicación $2012^{3}$. Los contenidos de esta fuente se complementaron y ampliaron durante el estudio con recursos específicos (como el directorio CRUE Gabinetes de Comunicación de las Universidades Españolas ${ }^{4}$ ), webs institucionales y otras fuentes electrónicas, con el objetivo de hallar los contactos más pertinentes para cada ocasión. Se combinaron pues los correos genéricos con otros más específicos, dirigidos a algunos profesionales individuales, puesto que como señala De-Bustos (2012, p. 223), actualmente el "gabinete de prensa debe tener al redactor como punto de partida de una red de difusión en la que ocupa el centro indiscutible. El periodista ha desplazado al medio como referencia del gabinete de prensa". También se difundieron las notas de prensa entre autores de blogs. En contraste con la posición mostrada por Dans en su artículo "Y el lugar de las notas de prensa... ¿qué?" ${ }^{5}$, creemos que los contenidos no sólo tienen un valor potencial para los medios, sino que las notas de prensa deben apuntar al espectro más amplio de

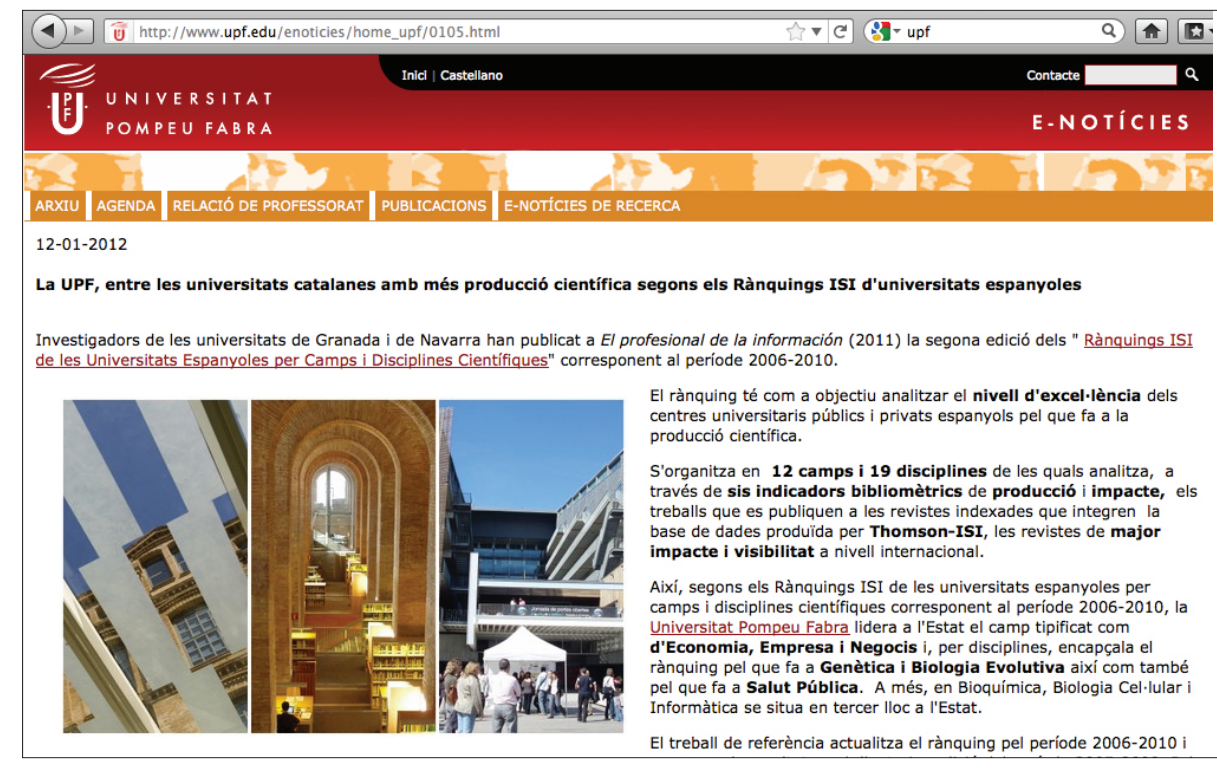

Figura 2. Universitat Pompeu Fabra

Tabla 2. Artículos de EPI utilizados en el experimento y número de impactos obtenido por cada artículo

\begin{tabular}{|c|c|}
\hline Artículo & Impactos \\
\hline $\begin{array}{l}\text { Tur-Viñes, Victoria; Fonseca-Mora, María-Carmen; Gutiérrez-San-Miguel, Begoña (2012). "Ética de la publicación científica: iniciati- } \\
\text { vas y recomendaciones". El profesional de la información, v. 21, n. 5, pp. 491-497. } \\
\text { http://dx.doi.org/10.3145/epi.2012.sep.07 }\end{array}$ & 5 \\
\hline $\begin{array}{l}\text { Torres-Salinas, Daniel; García-Moreno-Torres, José; Robinson-García, Nicolás; Delgado-López-Cózar, Emilio; Herrera, Francisco } \\
\text { (2011). “Rankings ISI de las universidades españolas según campos y disciplinas científicas (2ª ed. 2011)". El profesional de la información, } \\
\text { v. 20, n. 6, pp. 701-709. } \\
\text { http://dx.doi.org/10.3145/epi.2011.nov.16 }\end{array}$ & 4 \\
\hline $\begin{array}{l}\text { Hilera-González, José-Ramón; Fernández-Sanz, Luis; Suárez-Morón, Esther (2012). “Evaluación de la legibilidad de webs de univer- } \\
\text { sidades". El profesional de la información, v. 21, n. 5, pp. 468-474. } \\
\text { http://dx.doi.org/10.3145/epi.2012.sep.04 }\end{array}$ & 3 \\
\hline $\begin{array}{l}\text { Isasi-Andrieu, Alberto; López-Carrera, Ángel; Ruiz-Ibáñez, Pilar; Angulo-Redondo, Iñaki (2012). “Gazela: asesor digital de redes } \\
\text { sociales para adolescentes". El profesional de la información, v. 21, n. 5, pp. 514-519. } \\
\text { http://dx.doi.org/10.3145/epi.2012.sep.11 }\end{array}$ & 2 \\
\hline $\begin{array}{l}\text { Benito-Blánquez, Belén; Doñate-Cifuentes, Ana; Gómez-Domínguez, David; Marquina-Arenas, Julián; Martínez-Cañadas, Evelio } \\
\text { (2011)."Biblogtecarios: el poder de las conexiones y el crowdsourcing”. El profesional de la información, v. 20, n. 6, pp. 677-681. } \\
\text { http://dx.doi.org/10.3145/epi.2011.nov.12 }\end{array}$ & 0 \\
\hline $\begin{array}{l}\text { Blanco, Elena; Casaldàliga, Anna (2012). “Papel de la biblioteca en la difusión de la producción científica en la Universitat Pompeu } \\
\text { Fabra". El profesional de la información, v. 21, n. 6, pp. 627-631. } \\
\text { http://dx.doi.org/10.3145/epi.2012.nov.10 }\end{array}$ & 0 \\
\hline $\begin{array}{l}\text { Izquierdo-Castillo, Jessica (2012). “Distribución online de contenidos audiovisuales: análisis de } 3 \text { modelos de negocio”. El profesional } \\
\text { de la información, v. 21, n. 4, pp. 385-390. } \\
\text { http://dx.doi.org/10.3145/epi.2012.jul.09 }\end{array}$ & 0 \\
\hline $\begin{array}{l}\text { Larrondo, Ainara; Larrañaga-Zubizarreta, José; Meso, Koldo; Agirreazkuenaga, Irati (2012). “Convergencia de medios y redaccio- } \\
\text { nes: el caso de la Radio Televisión Pública Vasca (EITB)". El profesional de la información, v. 21, n. 4, pp. 347-353. } \\
\text { http://dx.doi.org/10.3145/epi.2012.jul.03 }\end{array}$ & 0 \\
\hline $\begin{array}{l}\text { Leydesdorff, Loet (2012). “Comparación y predicción de las cuotas mundiales de publicación de los EUA, EU-27 y China usando la } \\
\text { nueva interfaz Web of science versus Scopus”. El profesional de la información, v. 21, n. 1, pp. 43-50. } \\
\text { http://www.elprofesionaldelainformacion.com/leydesdorff-espanol.pdf }\end{array}$ & 0 \\
\hline $\begin{array}{l}\text { Martínez-Sanz, Raquel (2012). “Estrategia comunicativa digital en el museo”. El profesional de la información, v. 21, n. 4, pp. } 391-395 . \\
\text { http://dx.doi.org/10.3145/epi.2012.jul.10 }\end{array}$ & 0 \\
\hline
\end{tabular}




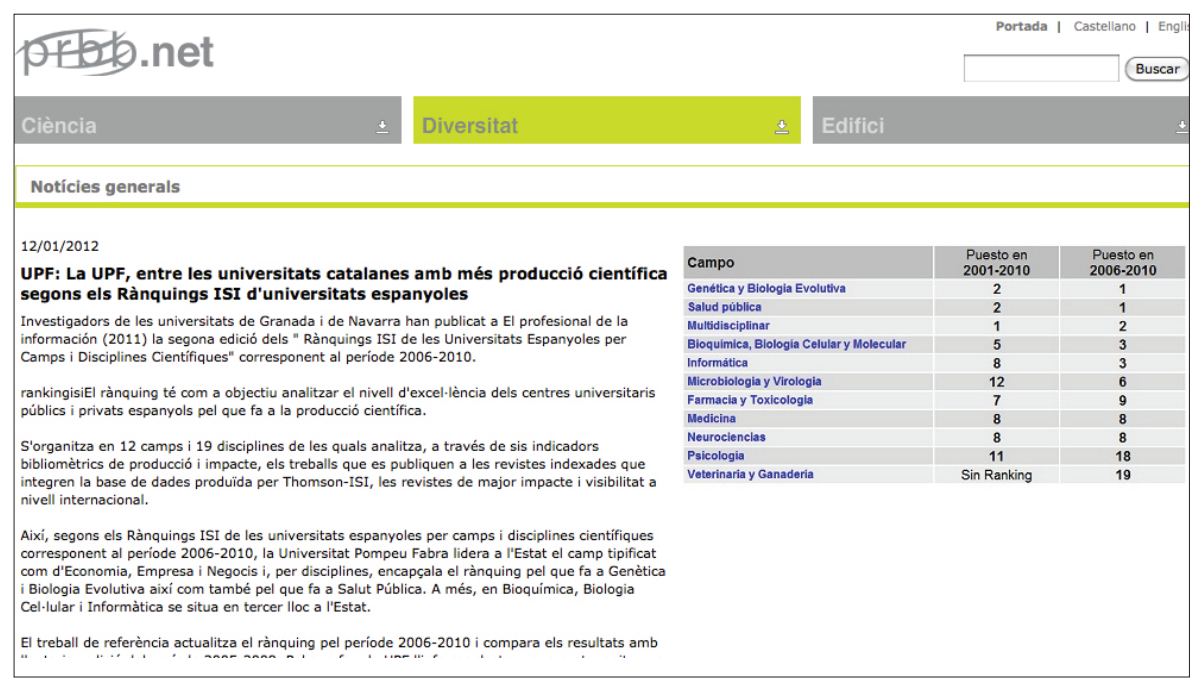

Figura 3. Parc de Recerca Biomèdica (PRBB)

representatividad respecto a los ejes temáticos de $E P I$, que se muestran en la tabla 2. Se trataron cuestiones referentes a la distribución audiovisual, bibliotecas, comunicación museística, convergencia mediática, redes sociales, producción y publicación científica o legibilidad web.

Cada pieza (la nota de prensa, en términos clásicos) se redactó siguiendo las características de la redacción periodística: estructuración en pirámide invertida, u orden lógico frente a orden cronológico; atención a las seis preguntas básicas ( 6 w questions); claridad, precisión, rigor, concreción y concisión en el texto y en los elementos de titulación (McCurry, 2004; Fernández-Asenjo; De-la-Torre-Alfaro, 2009; GarcíaOrosa, 2009; Foster, 2012; Pander-Maat, 2007; Pander-Maat; De-Jong, 2012; Risquete, 2006; Sissons, 2012). También se atendió a la naturaleza hipertextual de la redacción online (Díaz-Noci, 2002; Salaverría, 2005) y se acompañó cada difusión del artículo completo publicado en EPI en pdf.

La redacción de las notas informativas se rigió por los criterios éticos fundamentales de la profesión (Fernández-Asenjo; De-la-Torre-Alfaro, 2009; Risquete, 2006). Según explicita el Col-legi de Periodistes de Catalunya, "Ios gabinetes de comunicación prestan un servicio de información y de comunicación social, de acuerdo con las premisas deontoló-

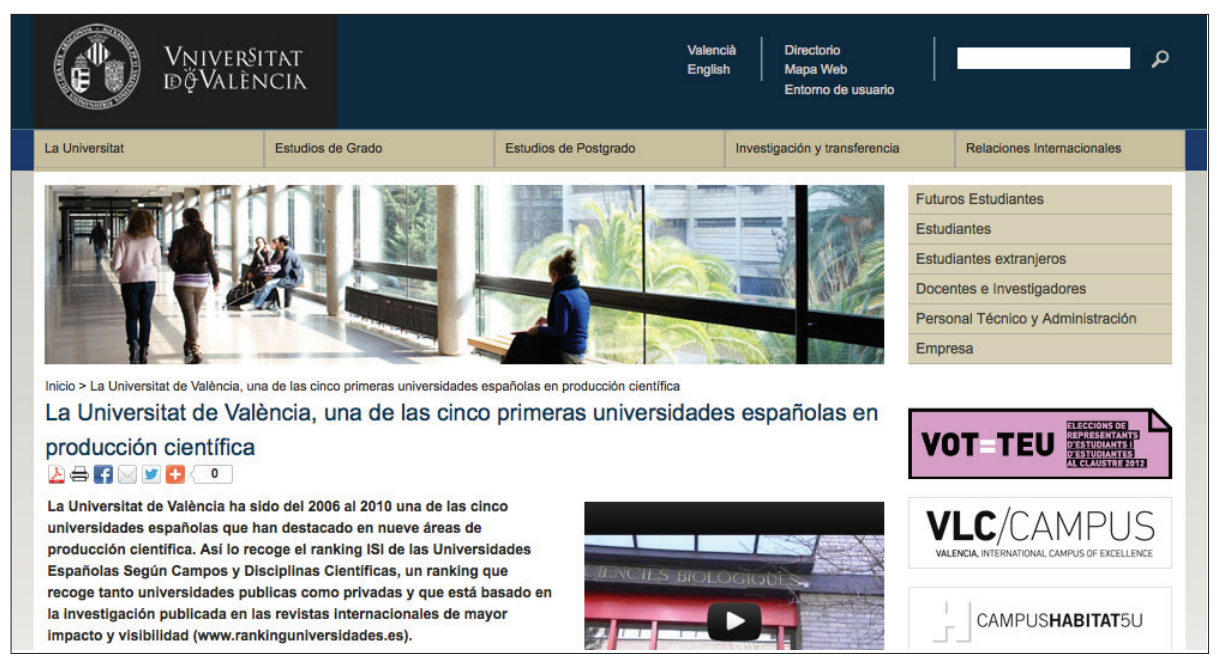

Figura 4. Universitat de València gicas del periodismo"6. Se marcaron los límites entre información y publicidad o marketing (De-Bustos, 2012; Foster, 2012), y se evitó la inclusión del lenguaje promocional descrito por Pander-Maat (2007, pp. 69-71), esto es, adjetivos y adverbios intensificadores, adjetivos valorativos o grados superlativos, entre otros. Asimismo se preservaron los principios de veracidad, libertad, justicia y responsabilidad social (Alsius, 2010; Frost, 2011), recogidos en los códigos éticos del periodismo promovidos por la Unesco, la Federación Internacional de Periodistas, el Consejo de Europa o la FAPE', así como en los documentos de referencia en las relaciones públicas, elaborados por la Public Relations Society of America $^{8}$ o la International Public Relations Association ${ }^{9}$.

El proceso de elaboración de cada nota (lectura y análisis del artículo original, adaptación y maquetación) y su distribución y envío (redacción y selección de los destinatarios relevantes para cada caso) requirió una dedicación aproximada de 3,5 horas por artículo. En el caso de las notas específicas o personalizadas, el tiempo utilizado fue superior (entre 5 y 6 horas).

\section{Resultados del experimento}

\subsection{Difusiones realizadas}

Se envió un total de 38 notas de prensa, correspondientes a 10 artículos publicados en El profesional de la información, aplicando varias estrategias de difusión:

se elaboraron notas de prensa con un único enfoque, basadas en siete artículos (a saber: Blanco; Casaldàliga, 2012; Benito-Blázquez et al., 2011; Isasi-Andrieu et al., 2012; Izquierdo-Castillo, 2012; Larrondo et al., 2012; Leydesdorff, 2012; Martínez-Sanz, 2012);

se realizaron notas personalizadas, como en el artículo "Ética de la publicación científica: iniciativas y recomendaciones" (Tur-Viñes; FonsecaMora; Gutiérrez-San-Miguel, 2012), del que se mandaron notas específicas a cada una de las universidades de las autoras. Esta estrategia también se refleja en otros artículos, como "Evaluación de la legibilidad de webs de universidades" (Hilera-González; Fernández-Sanz; Suárez-Morón, 2012), a partir del cual surgieron 6 notas: 1 pieza general y 5 notas específicas para los gabinetes de universidades (Alcalá de Henares, Barcelona, UNED, Pompeu Fabra y Politécnica de Madrid). A partir del artículo "Rankings ISI de las uni- 
versidades españolas según campos y disciplinas científicas (2 a ed. 2011)" (Torres-Salinas et al., 2012), se elaboró una nota personalizada para cada uno de los 21 centros recogidos en el estudio.

\subsection{Impactos obtenidos}

Se detectaron 14 impactos correspondientes a cuatro de los 10 temas mencionados anteriormente. Los seis restantes no obtuvieron impacto alguno. Según el impacto recibido:

1) el mejor resultado en términos de recepción fue para el artículo de Tur-Viñes; FonsecaMora; Gutiérrez-San-Miguel (2012). Los resultados científicos de este trabajo fueron recogidos por la Universidad de Salamanca ${ }^{10}$, Diariodesalamanca.es, Salamancauniversitaria.com, Salamanca24horas.com ${ }^{11}$ y la Agencia de Noticias para la Difusión de la Ciencia y la Tecnología $(D i C Y T)^{12}$.

\section{Cuatro artículos de El profesional de la} información generaron 14 impactos en medios de comunicación y plataformas institucionales

2) informaciones procedentes del artículo de Torres-Salinas et al. (2012), se reflejaron en las páginas de El Periòdic. Periódico digital de la Comunidad Valencia$n a^{13}$, Universitat Pompeu Fabra de Barcelona, Parc de Recerca Biomèdica $(P R B B)^{14}$ y Universitat de València ${ }^{15}$.

3) la nota del artículo "Evaluación de la legibilidad de webs de universidades" fue recogida por las páginas de la Universitat de Barcelona ${ }^{16}$ y de la Universidad de Alcalá de Henares ${ }^{17}$, así como en la página de ESVI-AL (Educación Superior Virtual Inclusiva-América Latina) ${ }^{18}$.

4) la nota "Gazela: asesor digital de redes sociales para adolescentes", elaborada a partir del artículo de Isasi-Andrieu et al. (2012), fue publicada en el periódico Deia.com ${ }^{19}$ y en el blog Ecommerce y Marketing ${ }^{20}$.

El número de impactos en medios de comunicación generalistas fue reducido en comparación con la repercusión en plataformas académicas. Cabe tener en cuenta la sobreabundancia de comunicados recibidos por los periodistas (FernándezAsenjo; De-la-Torre-Alfaro, 2009), que provoca que se deseche "el $85 \%$ de las notas de prensa que llegan a las redacciones" (De-Bustos, 2012, p. 226). Además, cabe considerar el poco espacio dedicado por los medios a la investigación, las escasas fuentes de las noticias científicas publicadas en la prensa española (Elías, 2000) y "la inseguridad del redactor sobre lo que es noticia científica relevante" (Elías, 2008, p. 84).

Tampoco se pueden obviar las dificultades técnicas. El envío de la primera nota de prensa subrayó una carencia muy significativa de la Agenda de la Comunicación. Entre las 1.120 direcciones que integraron el primer listado de mailing, se obtuvo una tasa de correos rechazados del $10 \%$. Se observaron como causas principales de estos rechazos los buzones de correo llenos y direcciones desactualizadas. Esta cifra

\section{uah esnoticia}

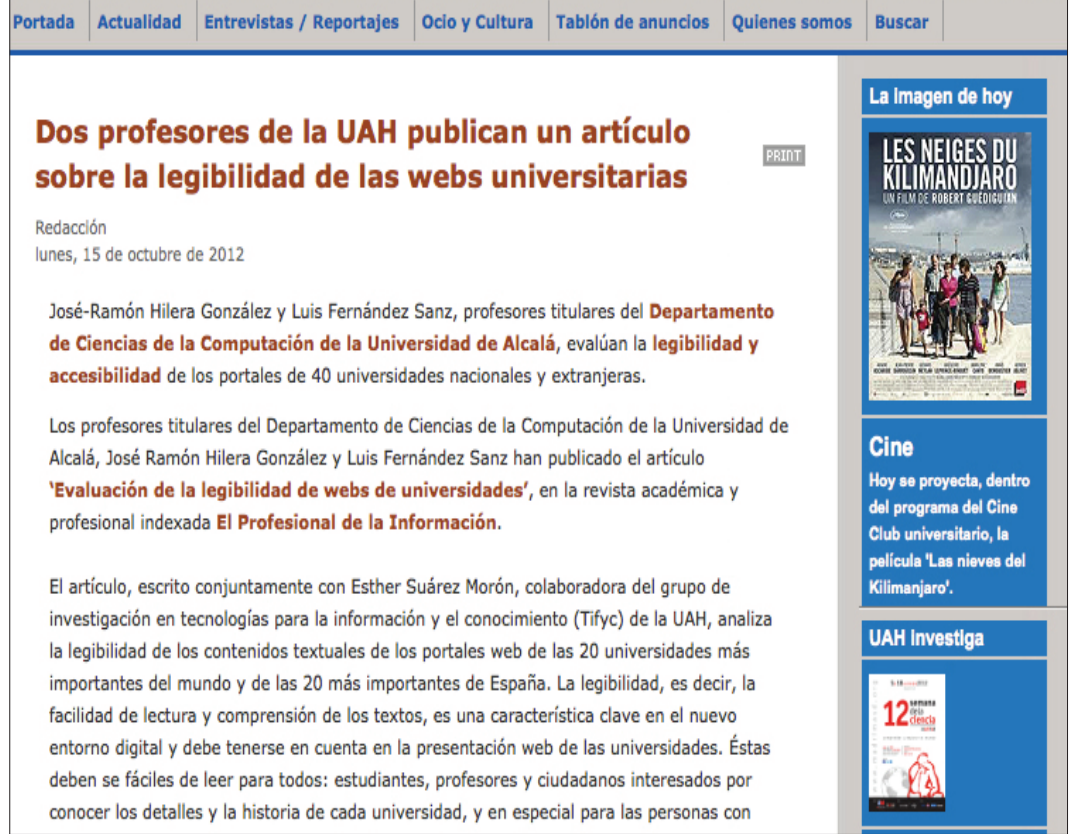

Figura 6. Universidad de Alcalá de Henares 


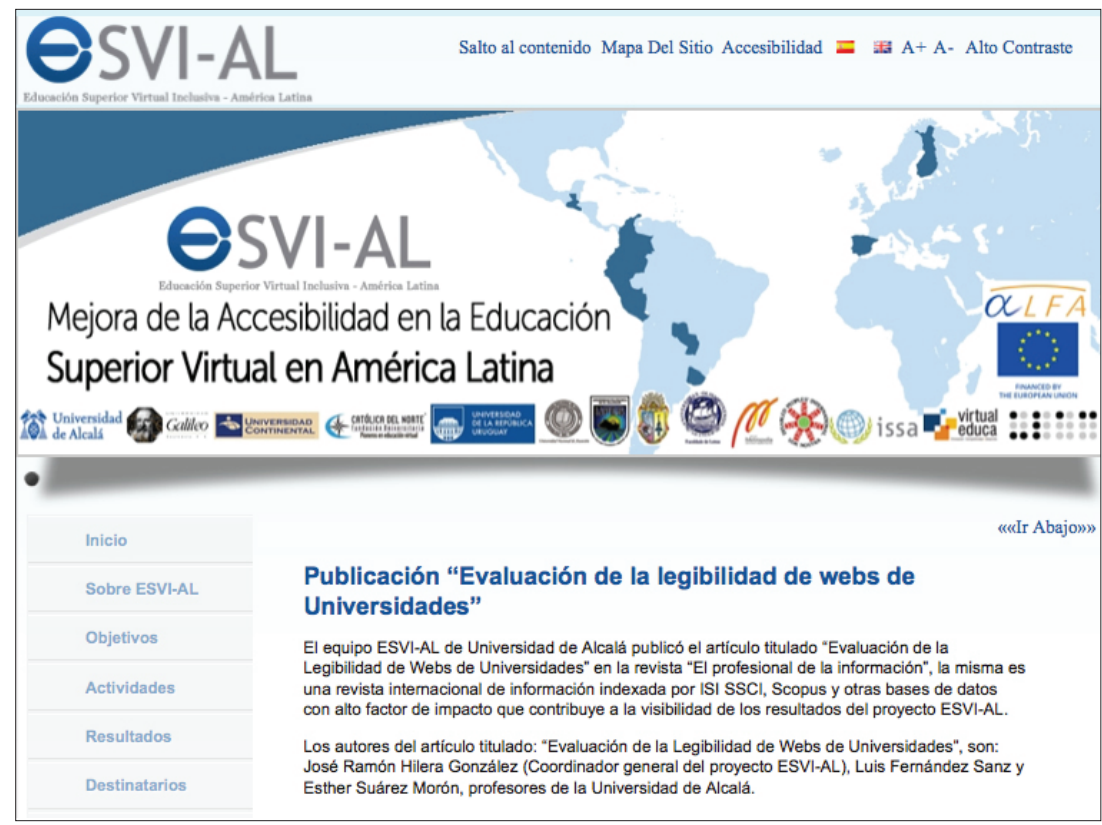

Figura 7. Educación Superior Virtual Inclusiva - América Latina (ESVI-AL)
Todos los textos mencionaron a los autores de los artículos y su filiación, a excepción de la $U B$, que no referenció a una de las autoras del artículo sobre legibilidad de webs. Pese a todo, el título exacto del artículo publicado en $E P I$ sólo se reflejó en nueve de catorce ocasiones y sólo en los casos de UPF y $P R B B$ se incluyó la referencia bibliográfica completa.

Los enlaces a la revista o al resumen del artículo en la página web de la misma fueron escasos (UPF, PRBB, UB y ESVI$A L)$. Cabe añadir que sólo dos piezas (Deia.com y ESVI-AL) incluyeron citas entrecomilladas con contenido de los artículos científicos.

Finalmente, no se detectaron incorrecciones en la información transmitida, a excepción de un leve error (UPF) en que se señaló a "Thomson-ISI" como productor de la base de datos, cuando hamanifiesta el grado de obsolescencia de un documento de referencia para el sector comunicativo español. En la Agenda de la Comunicación 2012 se detectaron carencias similares, puesto que se incluían empresas que habían cesado su actividad o habían cambiado de denominación.

\subsection{Características de los impactos obtenidos}

Mediante la aplicación de la técnica del análisis del contenido (Bryman, 2012; Krippendorff, 2004; Ruiz-Olabuénaga; Aristegui; Melgosa, 1998), se estudiaron las piezas publicadas y se alcanzaron los siguientes resultados:

Se puede apreciar una ligera variación en cuanto al número de palabras del texto original. Si bien las notas enviadas desde EPI tenían una extensión media de 403 palabras, esta cifra ascendió hasta las 459 palabras en los materiales publicados. Sin embargo, no se produjeron grandes cambios a nivel de contenido editorial, pues se respetó el sentido y el fondo de la información transmitida. En determinados casos, como en el texto de la Universidad de Salamanca, el incremento de palabras respecto a la nota enviada (de 421 a 644) se acompañó de una mayor profundización en el artículo original y de información docente e investigadora de la autora. En otras ocasiones la traslación de contenidos fue casi exacta, lo que reforzó la idea de que en lugar de editar nuevamente la información, se replicó prácticamente el material proporcionado (Sissons, 2012).

La circulación libre y eficaz de los contenidos científicos es uno de los principales deberes de las sociedades democráticas modernas

En todos los casos se citó a El profesional de la información como fuente, aunque sólo ESVI-AL añadió más datos contextuales sobre la revista (índices y factor de impacto). blando con propiedad, la base de datos ISI Web of knowledge es editada por Thomson-Reuters.

\section{Conclusiones y líneas futuras de investigación}

Como se ha explicado, la muestra se ha circunscrito a una revista, un ámbito científico y un período temporal concreto. Por consiguiente, no es posible extrapolar los resultados hacia otros contextos. Sin embargo, aquellos aportan datos de interés que permiten apuntar a futuras investigaciones.

Se respetó el sentido y fondo de la información, pero en la mayoría de los casos simplemente se replicó el material proporcionado y se demostró una escasa profundización en el artículo original

Los resultados obtenidos hasta el momento indican la pertinencia de ahondar en la estrategia de realización de notas de prensa, con el objetivo de contribuir a una mayor visibilidad de los contenidos de la revista. Las notas de prensa deben seguir jugando un papel fundamental en la divulgación de la actividad científica. Una cuidada adaptación de los artículos se considera clave para una posterior difusión completa y que respete la información relevante y las fuentes utilizadas en cada trabajo. Para ello es recomendable que las revistas cuenten con un equipo de colaboradores que diseñe y aplique una estrategia de difusión de contenidos, que vele por la calidad de los mismos y que ahonde en la relación con los destinatarios de la información, por teléfono y encuentros personales, si es posible, ya que la interacción y la recepción de feedback (García-Orosa, 2009; Ros-Martín, 2008) también puede resultar beneficiosa para mejorar la calidad de los contenidos (Escribà; Cortiñas-Rovira, 2013).

Además conviene seguir potenciando el uso de las redes sociales ${ }^{21}$. Las revistas no pueden infravalorar los beneficios 
de Twitter, utilizado de forma creciente como herramienta por parte de los profesionales para aproximarse y citar a las fuentes (Broersma; Graham, 2013). La capacidad de adaptarse a los nuevos canales y determinar su utilidad para la difusión científica deviene una tarea de primer orden.

Estas cuestiones señalan algunos retos futuros de investigación. En primer lugar, se debe seguir trabajando y monitorizando sobre las notas de El profesional de la información. En segundo lugar, sería relevante realizar el mismo experimento con diferentes revistas de impacto a nivel nacional e internacional, con el fin de analizar y comparar las estrategias de difusión y los impactos obtenidos. En nuevos experimentos, se debería potenciar la difusión de artículos en las redes sociales generalistas y plataformas académicas especializadas (Academia. edu y ResearchGate.com). Por último sería pertinente triangular (Tashakkori; Teddlie, 2009) la metodología experimental con entrevistas en profundidad (Marshall; Rossman, 2011; Rubin; Rubin, 1995) con autores, miembros de consejos editoriales de revistas y receptores de la información científica (investigadores, profesorado, periodistas, profesionales de los sectores relevantes, ciudadanos), para conocer mejor las expectativas de producción y recepción de los materiales. De este modo en futuros trabajos también se podría examinar si las acciones realizadas se traducen en un incremento de las suscripciones a dichas publicaciones.

Y es que más allá de las voluntades puramente promocionales de las revistas o de las entidades editoras, está en juego uno de los principales deberes de las sociedades democráticas modernas: la circulación libre y diligente de la investigación puntera de calidad, obtenida a través del método científico, uno de los mayores patrimonios que el conocimiento ha dado en todos los tiempos. Estamos todavía lejos de que el costoso camino que va de la comunidad científica especializada hacia el gran público sea firme y duradero; y sea, a su vez, transitado por los numerosos actores implicados de forma frecuente y eficaz.

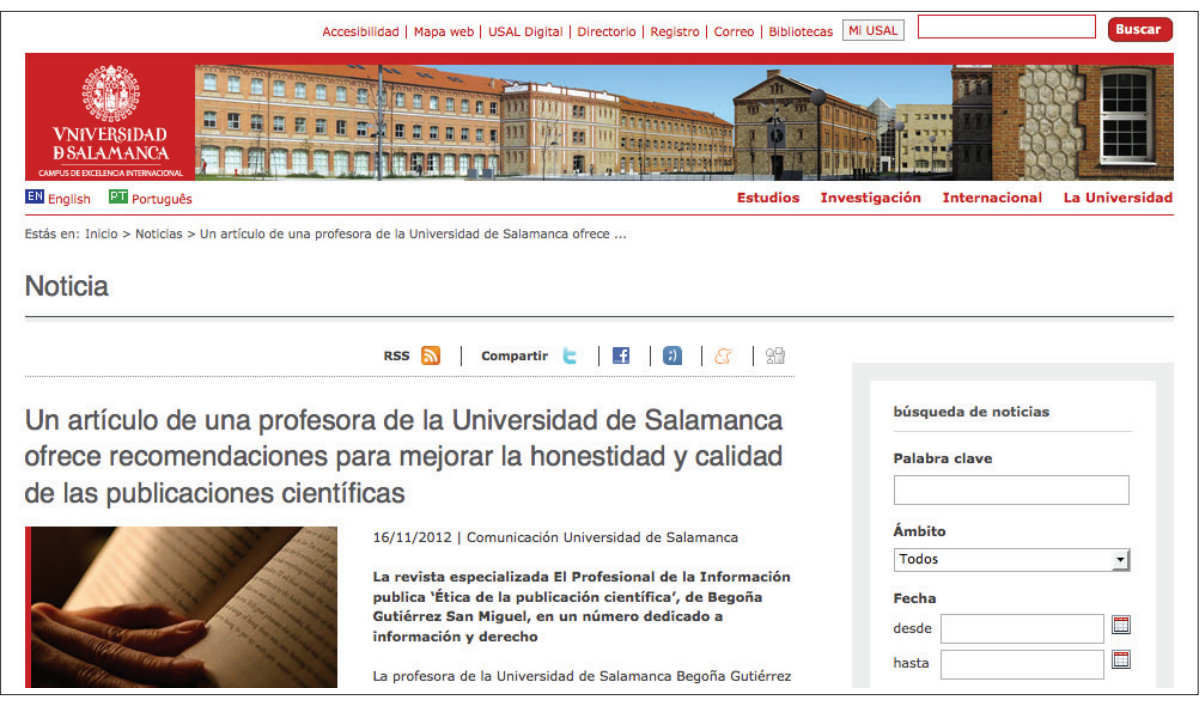

Figura 8. Universidad de Salamanca

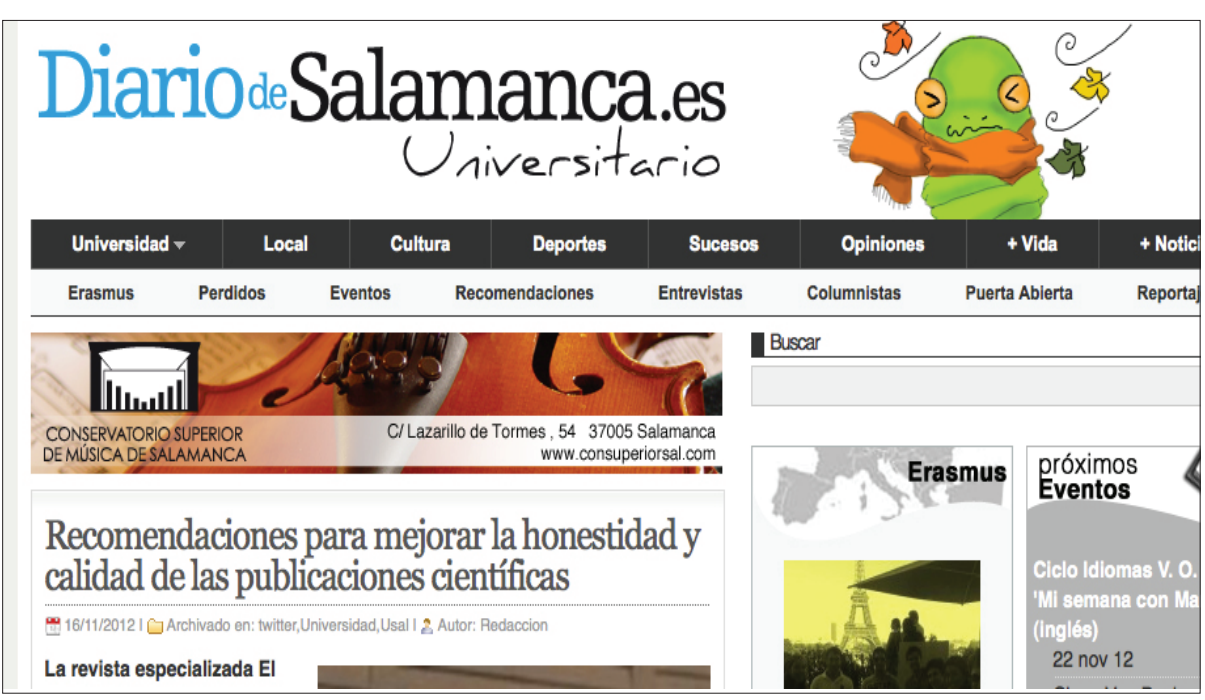

Figura 9. Diario de Salamanca

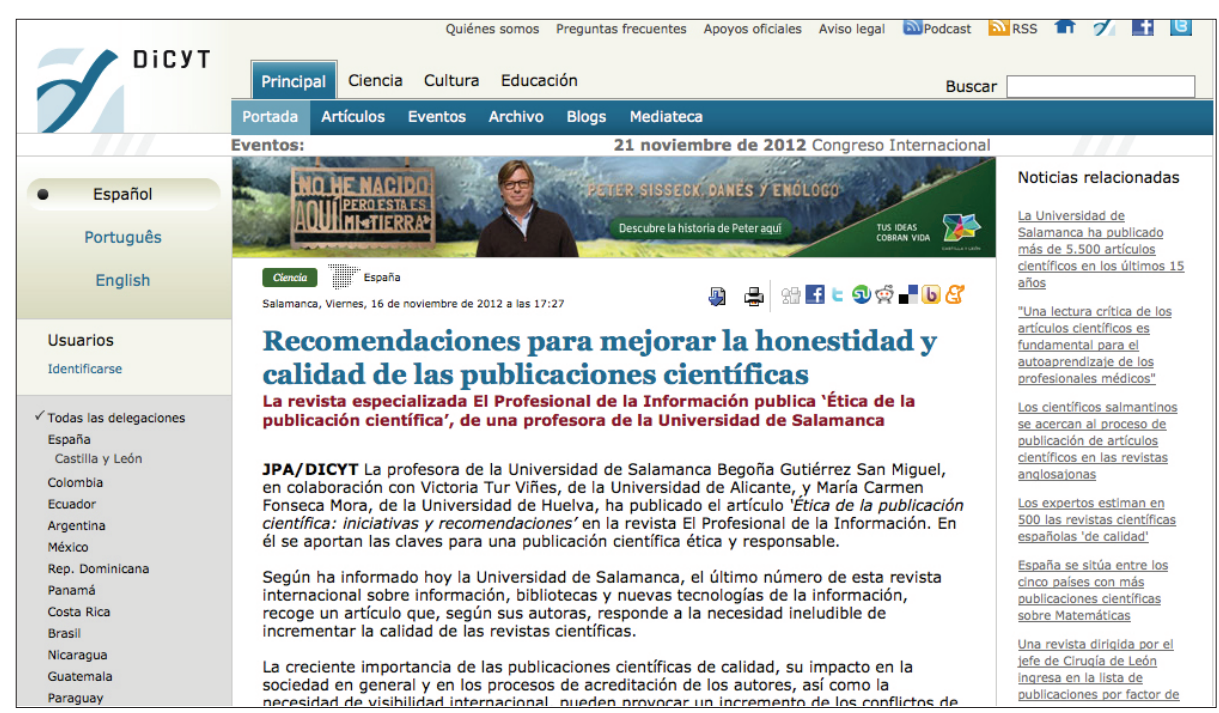

Figura 10. Agencia de Noticias para la Difusión de la Ciencia y la Tecnología (DiCYT).

\section{Notas}

1. Agenda de la comunicación 2011

http://es.scribd.com/doc/51112123/Agenda-de-laComunicacion-2011-Espana-Ministerio-de-la-PresidenciaSecretaria-de-Estado-de-Comunicacion-Espana 


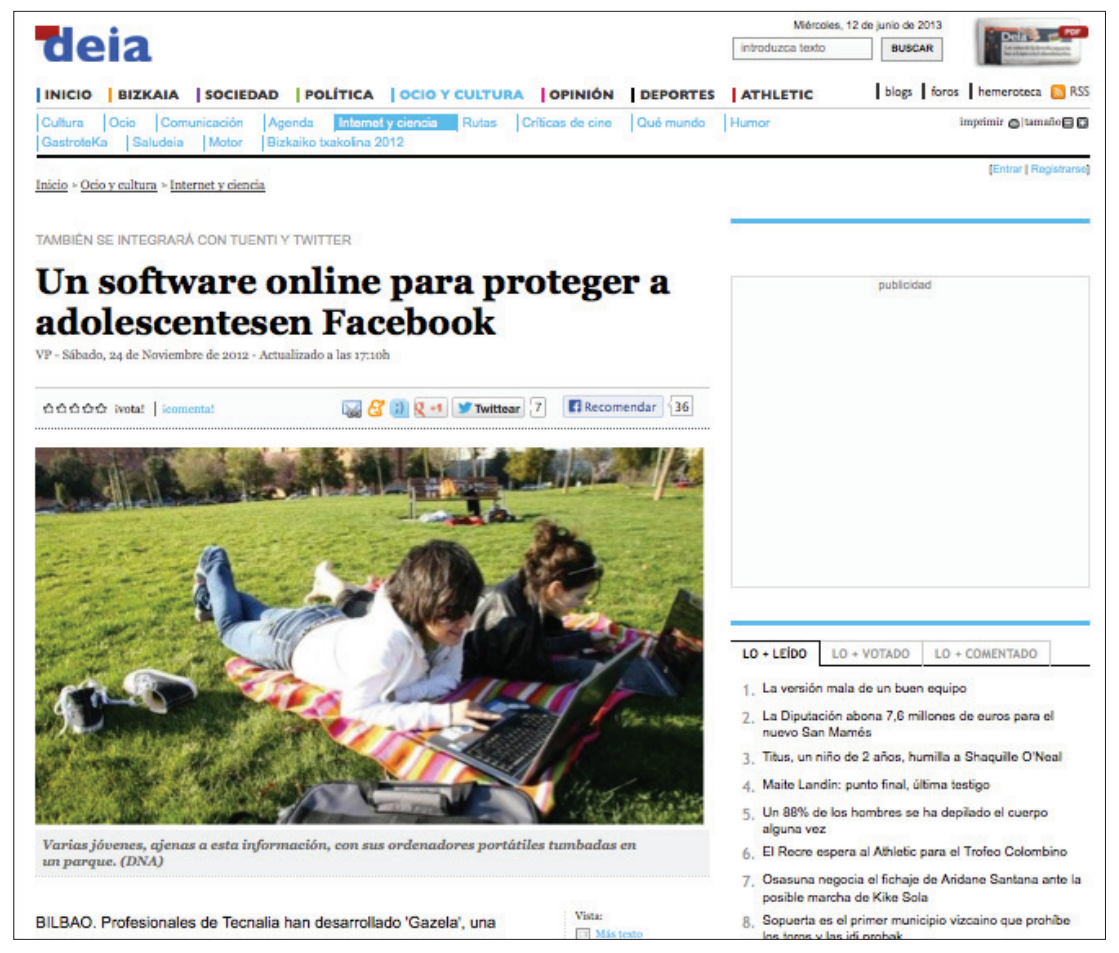

Figura 11. Deia.com

2. Para conducir el experimento se descartó el uso del correo postal. Se valoró la visibilidad que se puede llegar a obtener con este método, pero se consideraron sus inconvenientes, como la dificultad de llegar a cada receptor de forma directa, rápida y eficiente o la imposibilidad de aportar elementos de valor añadido, como los enlaces, materiales en otros soportes o el texto electrónico, que posteriormente puede ser copiado, adaptado y refundido.

3. Agenda de la comunicación 2012 http://www.lamoncloa.gob.es/NR/rdonlyres/120271BF7C97-422E-B6B0-3938B9BE2E5F/0/Agenda2012.pdf

4. Listado de gabinetes de comunicación de las universidades españolas:

http://www.crue.org/prensa/documentos/Gabinetes_ Universidades_Espanolas_marzo2013.pdf

5. Dans, Enrique (2012). "Y el lugar de las notas de prensa... ¿qué?". El blog de Enrique Dans, 27 de diciembre.

http://www.enriquedans.com/2012/12/y-en-lugar-de-lasnotas-de-prensa-que.html

6. Col/legi de Periodistes de Catalunya. "Els gabinets de comunicació. Criteris de bones pràctiques professionals".

http://www.periodistes.org/fcic/admin/doc_contingut/ MANUAL_BONES_PRACTIQUES.pdf

7. Recopilación de códigos éticos del periodismo realizada por los profesores Kaarle Nordenstreng y Ari Heinonen de la University of Tampere (Finlandia).

http://ethicnet.uta.fi/codes_by_country

8. Public Relations Society of America (PRSA) "Member code of ethics".

http://www.prsa.org/AboutPRSA/Ethics/documents/ Code\%20of\%20Ethics.pdf

9. International Public Relations Association (IPRA). "Code of conduct".

http://www.ipra.org/about/ipra-codes

10. "Un artículo de una profesora de la Universidad de Salamanca ofrece recomendaciones para mejorar la honestidad y calidad de las publicaciones científicas". Universidad de Salamanca. 16/11/2012.

http://www.usal.es/webusal/ node/26597

11. La dirección de la pieza publicada por Diariodesalamanca.es no está disponible. En las siguientes direcciones se pueden encontrar las otras notas refundidas:

http://www.salamancauniversitaria. com/usal/906-recomendaciones-paramejorar-la-honestidad-y-calidad-de-laspublicaciones-cientificas

http://www.salamanca24horas.com/ local/77343-recomendaciones-paramejorar-la-honestidad-y-calidad-de-laspublicaciones-cientificas

12. "Recomendaciones para mejorar la honestidad y calidad de las publicaciones científicas". DiCYT, $16 / 11 / 2012$

http://www.dicyt.com/noticias/recomendaciones-paramejorar-la-honestidad-y-calidad-de-las-publicacionescientificas

13. "La Universitat de València, una de las cinco primeras universidades españolas en producción científica". El Periòdic. Periódico digital de la Comunidad Valenciana. 28/01/2012.

http://www.elperiodic.com/pvalencia/noticias/156101_ universitat-valencia-cinco-primeras-universidadesespaolas-produccion-cientifica.html

14. Debido a la migración a la nueva web de la Universitat Pompeu Fabra llevada a cabo a principios de septiembre de 2012, estas direcciones ya no están disponibles.

15. "La Universitat de València, una de las cinco primeras universidades españolas en producción científica". Universitat de València. 26/01/2012.

http://www.uv.es/uvweb/universidad/es/lista-noticias/ universitat-valencia-cinco-primeras-universidadesespanolas-produccion-cientifica-1285846070123/Noticia. html?id=1285849964442

16. "La UB, entre las mejor posicionadas en el 'ranking' de legibilidad de las webs universitarias españolas". Universitat de Barcelona, 19/10/2012.

http://www.ub.edu/web/ub/es/menu_eines/ noticies/2012/10/091.html

17. "Dos profesores de la UAH publican un artículo sobre la legibilidad de las webs universitarias". Universidad de Alcalá de Henares, 15/10/2012.

http://www2.uah.es/diariodigital/index 2 . php?option=com_content $\&$ task $=v i e w \& i d=6449$

18. “Publicación 'Evaluación de la legibilidad de webs de 
Universidades'”. Educación Superior Virtual Inclusiva-América Latina (ESVI-AL).

http://www.esvial.org/?p=678

19. "Un software online para proteger a adolescentes en Facebook". Deia.com. 24/11/2012.

http://www.deia.com/2012/11/24/ocio-y-cultura/internet/unsoftware-online-para-proteger-a-adolescentesen-facebook

20. "Tecnalia ha desarrollado Gazela, una plataforma online para proteger a adolescentes en el uso de las redes sociales". Ecommerce y marketing, 26/11/2012.

http://ecommerceymarketing.blogspot.com.es/2012/11/ tecnalia-ha-desarrollado-gazela-una.html

21. Durante el estudio, los investigadores no difundieron el contenido de las notas de prensa o de los artículos originales en las redes sociales.

Las revistas deben contar con una estrategia de difusión de contenidos, potenciar las redes sociales y ahondar en la relación con los destinatarios de la información

\section{Bibliografía}

Almansa-Martínez, Ana (2011). Del gabinete de prensa al gabinete de comunicación. La dirección de comunicación en la actualidad. Zamora: Comunicación Social. ISBN: 9788492860906

Alsius, Salvador (ed.) (2010). The ethical values of journalists. Field research among media professionals in Catalonia. Barcelona: Generalitat de Catalunya. ISBN: 9788439383468

Baiget, Tomàs; Torres-Salinas, Daniel (2013). Informe APEI sobre publicación en revistas científicas. Gijón: Asociación Profesional de Especialistas en Información.

http://www.apei.es/informes/InformeAPEI-Publicacionescientificas.pdf

Baines, Paul; Egan, John; Jefkins, Frank (2004). Public relations: contemporary issues and techniques. Oxford: Elsevier Butterworth-Heinemann. ISBN: 0750657243

Broersma, Marcel; Graham, Todd (2013). "Twitter as a news source. How Dutch and British newspapers used tweets in their news coverage, 2007-2011". Journalism practice, publicado online 30 de mayo, pp. 1-19.

http://dx.doi.org/10.1080/17512786.2013.802481

Bryman, Alan (2012). Social research methods (4 ${ }^{\text {th }}$ ed.). Oxford: Oxford University Press. ISBN: 9780199588053

Campbell, Donald T.; Stanley, Julian C. (1966). Experimental and quasi-experimental designs for research. Boston: Houghton Mifflin Company. ISBN: 9780395307878.

Col-legi de Periodistes de Catalunya (2009). Els gabinets de comunicació. Criteris de bones pràctiques professionals. Barcelona: Col-legi de Periodistes de Catalunya.

http://www.periodistes.org/fcic/admin/doc_contingut/ MANUAL_BONES_PRACTIQUES.pdf
De-Bustos, Rosa (2012). "El gabinete de prensa ante el nuevo ecosistema informativo digital". adComunica. Revista científica de estrategias, tendencias e innovación en comunicación, n. 4, pp. 223-227.

http://repositori.uji.es/xmlui/bitstream/handle/10234/53579/78127-1-PB.pdf?sequence $=1$

http://dx.doi.org/10.6035/2174-0992.2012.4.14

De-Semir, Vladimir; Ribas, Cristina; Revuelta, Gemma (1998). "Press releases of science journal articles and subsequent newspaper stories on the same topic". Journal of the American Medical Association, v. 280, n. 3, pp. 294-295. http://jama.jamanetwork.com/article. aspx?articleid=187769 http://dx.doi.org/10.1001/jama.280.3.294

Díaz-Noci, Javier (2002). La escritura digital: hipertexto y construcción del discurso informativo en el periodismo electrónico. Bilbao: Servicio Editorial de la Universidad del País Vasco. ISBN: 8483733781

Domínguez-Romero, Martí (2002). "Divulgar la investigación con revistas científicas: el caso de Mètode". Comunicar. Revista científica de comunicación y educación, v. 19, pp. 49-53.

http://www.revistacomunicar.com/indice/articulo. php?numero=19-2002-09

Elías, Carlos (2000). Flujos de información entre científicos y prensa. Tesis doctoral, Universidad de La Laguna. ftp://tesis.bbtk.ull.es/ccssyhum/cs194.pdf

Elías, Carlos (2002). "Influencia de las revistas de impacto en el periodismo científico y en la ciencia actual". Reis. Revista española de investigaciones sociológicas, n. 98, pp. 123-137. http://www.redalyc.org/articulo.oa ?id $=99717918007$

Elías, Carlos (2008). “El periodismo científico como paradigma de la 'noticia acatamiento'. Una demostración desde las fuentes y una alerta de sus peligros". Periodística, n. 11, pp. 81-93. http://www.raco.cat/index.php/Periodistica/article/ view/245699

Escribà, Eudald; Cortiñas-Rovira, Sergi (2013). “Internationalization and coauthorship in major communication journals in Spain". Comunicar, v. XXI, n. 41.

http://dx.doi.org/10.3916/C41-2013-03

Fernández-Asenjo, Gerardo; De-la-Torre-Alfaro, Noa (2009). Gabinetes de comunicación en mínimos. Todos los porqués sobre las funciones y disfunciones de los gabinetes pequeños y con pocos recursos. Madrid: Fragua. ISBN: 978 8470743375

Foster, John (2012). Writing skills for public relations: style and technique for mainstream and social media. London: Kogan Page Publishers. ISBN: 9780749465438

Frost, Chris (2011). Journalism ethics and regulation (3 $3^{\text {rd }}$ ed.). Londres: Longman. ISBN: 9781408244685

García-Orosa, Berta (2009). Gabinetes de comunicación on line. Claves para generar información corporativa en la Red. Sevilla / Zamora: Comunicación Social. ISBN: 9788496082786

Krippendorff, Klaus (2004). Content analysis. An introduction to its methodology ( $2^{\text {nd }}$ ed.). Thousand Oaks, California: Sage, 2004. ISBN: 0761915443 
Loeffler, Robert H. (1993). A guide to preparing cost-effective press releases. Binghamton (New York): The Haworth Press, 1993. ISBN: 1560241411

Marshall, Catherine; Rossman, Gretchen B. (2011). Designing qualitative research ( $5^{\text {th }}$ ed.). Thousand Oaks, California: Sage, 2011. ISBN: 9781412970440

McCurry, Mike (2004). Media relations handbook: for agencies, associations, nonprofits and congress. Alexandria, Virginia (USA): The Capitol Net Inc. ISBN: 1587330032

Nieto, Josep; Vendrell, Montserrat (2001). "Els gabinets de premsa com a mediadors entre els científics i els mitjans". Treballs de la Societat Catalana de Biologia, v. 51, pp. 159-165. http://revistes.iec.cat/index.php/TSCB/article/ view/6557/60292

Pander-Matt, Henk (2007). "How promotional language in press releases is dealt with by journalists. Genre mixing or genre conflict?". Journal of business communication, v. 44, n. 1, pp. 59-95. http://dx.doi.org/10.1177/0021943606295780

Pander-Maat, Henk; De-Jong, Caro (2012). "How newspaper journalists reframe product press release information". Journalism, v. 14, n. 3, pp. 348-371.

http://dx.doi.org/10.1177/1464884912448914

Priest, Susanna-Hornig (2009). Doing media research: an introduction. Thousand Oaks, California, Sage. ISBN: 978 1412960977

Risquete, Jaume (2006). "Gabinetes de premsa i de comunicació. L'ambigua frontera entre el periodisme i les relacions públiques". Anàlisi, n. 34, pp. 209-221.

Ros-Martín, Marcos (2008). “Metodología para la implementación de un blog corporativo externo". El profesional de la información, v. 17, n. 5, pp. 502-509.

http://www.elprofesionaldelainformacion.com/ contenidos/2008/septiembre/03.pdf http://dx.doi.org/10.3145/epi.2008.sep.03

Rubin, Herbert J.; Rubin, Irene S. (1995). Qualitative interviewing. The art of hearing data. Thousand Oaks, California: Sage. ISBN: 9780803950962

Ruiz-Olabuénaga, José I.; Aristegui, Iratxe; Melgosa, Leire (1998). Cómo elaborar un proyecto de investigación social. Bilbao: Universidad de Deusto. ISBN: 9788474855470

Salaverría, Ramón (2005). Redacción periodística en internet. Pamplona: Ediciones Universidad de Navarra (Eunsa). ISBN: 9788431322595

Sissons, Helen (2012) "dournalisme and publich relations: a tale of two discourses". Discourse \& communication, v. 6, n. 3, pp. 273-294.

http://dx.doi.org/10.1177/1750481312452202

Tashakkori, Abbas; Teddlie, Charles (2009). Foundations of mixed methods research: integrating quantitative and qualitative approaches in the social and behavioral sciences. Thousand Oaks, California: Sage. ISBN: 9780761930112

Webster, Murray; Sell, Jane (2007). Laboratory experiments in the social sciences. Burlington, Massachusetts: Academic Press. ISBN: 9780123694898

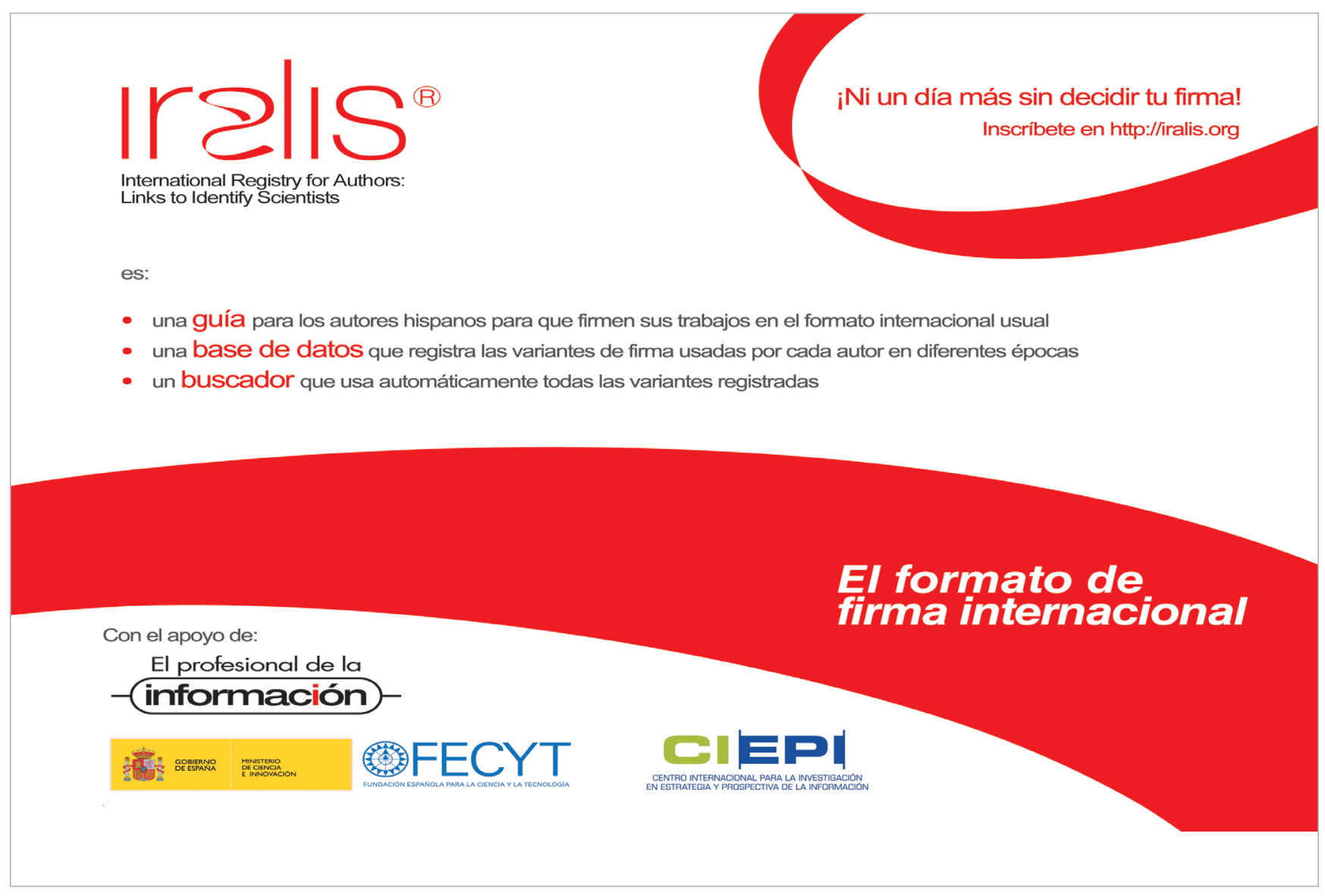

